\title{
Characterization of Semiconductor Materials Using Electron Holography
}

\author{
Lin Zhou ${ }^{1}$, Zhaofeng Gan², Myung-Geun Han ${ }^{3}$, David J. Smith ${ }^{4}$ and Martha R. McCartney ${ }^{4}$ \\ 1. Ames Laboratory, Ames, IA 50014, USA \\ 2. Intel Corporation, Hillsboro, OR 97124, USA \\ 3. Brookhaven National Laboratory, Upton, NY 11973, USA \\ 4. Department of Physics, Arizona State University, Tempe, AZ 85287, USA
}

Off-axis electron holography in the transmission electron microscope provides a unique and powerful approach for visualizing electric and magnetic fields within materials with resolutions approaching the nanometer scale. This ability to image phase shifts at medium resolution, and to quantify the corresponding electrostatic profiles, has opened up a wide range of interesting and important semiconductor materials problems for study, particularly in the area of electronic devices where other characterization techniques can no longer be used because of limited resolution [1]. This experimental capability has proven to be invaluable when developing strategies for doping semiconductor devices or when carrying out device modeling and simulations. However, minimizing unwanted artifacts resulting from sample preparation, such as implanted ions or amorphization of surface layers during focused-ionbeam milling, remains an ongoing challenge that must always be addressed when using the technique.

Figure 1 shows an example where off-axis electron holography has been used for quantitative analysis of the 2-D electrostatic potential distributions in 90-nm Si p-channel MOSFETs [2]. The measured electrostatic potentials from two $p$-MOSFETs with different offset spacer oxide widths were compared in terms of the detailed shape of extension junctions and source/drain (S/D) junctions, and it was found experimentally that the lateral drain potential profile was twice as abrupt as the vertical drain potential. Further studies of electrostatic fields in semiconductor materials include the imaging of phase shifts at quantum dots, which allow for quantification of charge capture, and the measurement of piezoelectric fields and 2-dimensional electron gas (2DEG) densities. As an example, Figures 2(a) and (b) show phase and amplitude images, respectively, from the reconstructed hologram of an AlInN/AlN/GaN high electron mobility transistor (HEMT). Profile measurements, shown in Fig. 2(c), indicate a polarizationinduced electric field of $6.9 \mathrm{MV} / \mathrm{cm}$ within the AlN layer, and 2DEG with a density of $\sim 2.1 \times 10^{13} \mathrm{~cm}^{-2}$ located in the GaN about $0.8 \mathrm{~nm}$ away from the AlN/GaN interface [3]. The holography technique is also suitable for studying changes in potential profiles as a result of in situ biasing. As an example, we have used off-axis electron holography to measure charge distribution during lithium ion insertion into a Ge nanowire (NW) under dynamic operating conditions [4]. Figures 3(a) and (d) are sequential holograms recorded while lithiation was taking place, while Figures 3(b) and (e) show the corresponding phase images. Profile measurements, combined with simulations, enabled the Li component of the NW to be estimated and the trapped charge to be quantified.

[1] M.R. McCartney and D.J. Smith, Annu. Rev. Mater. Sci. 37 (2007) p.729

[2] M.-G. Han, et al., IEEE Trans. Electron Devices, 54 (2007) p. 3336.

[3] L. Zhou et a.l, Appl. Phys. Lett., 94, (2009), 121909.

[4] Z. Gan, et al., Nano Lett., 16 (2016) p. 3748.

[5] The authors acknowledge the use of facilities in the John M. Cowley Center for High Resolution

Electron Microscopy at Arizona State University. 

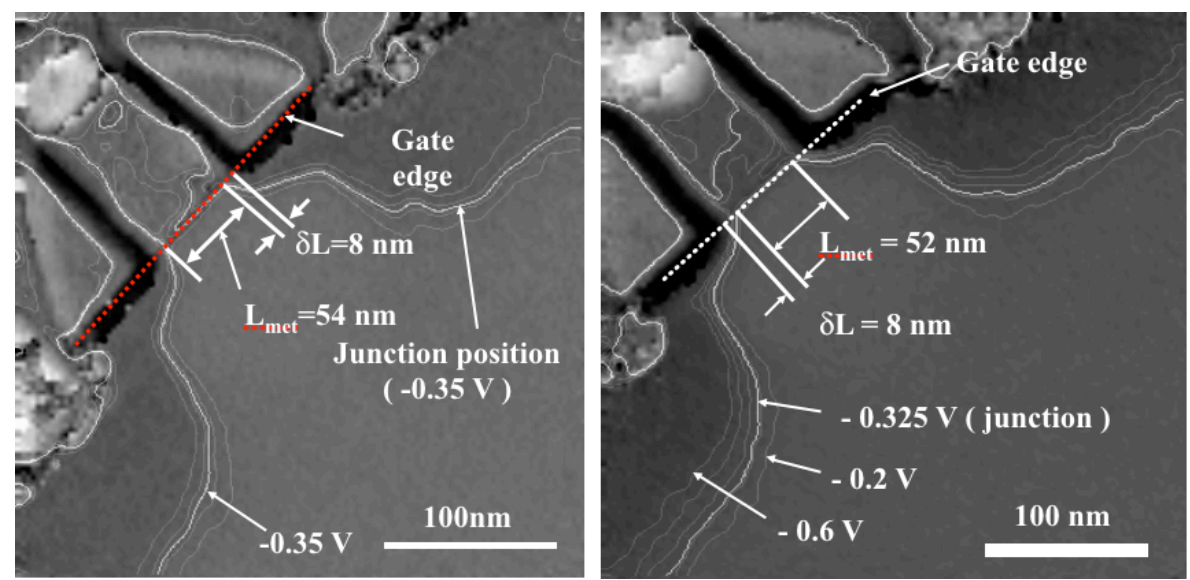

Figure 1. 2-D electrostatic potential contour maps in 90-nm Si p-MOSFET device: (L) 0-nm, and (R) 14-nm oxide spacer. Contour separation of $0.1 \mathrm{~V}[1]$.
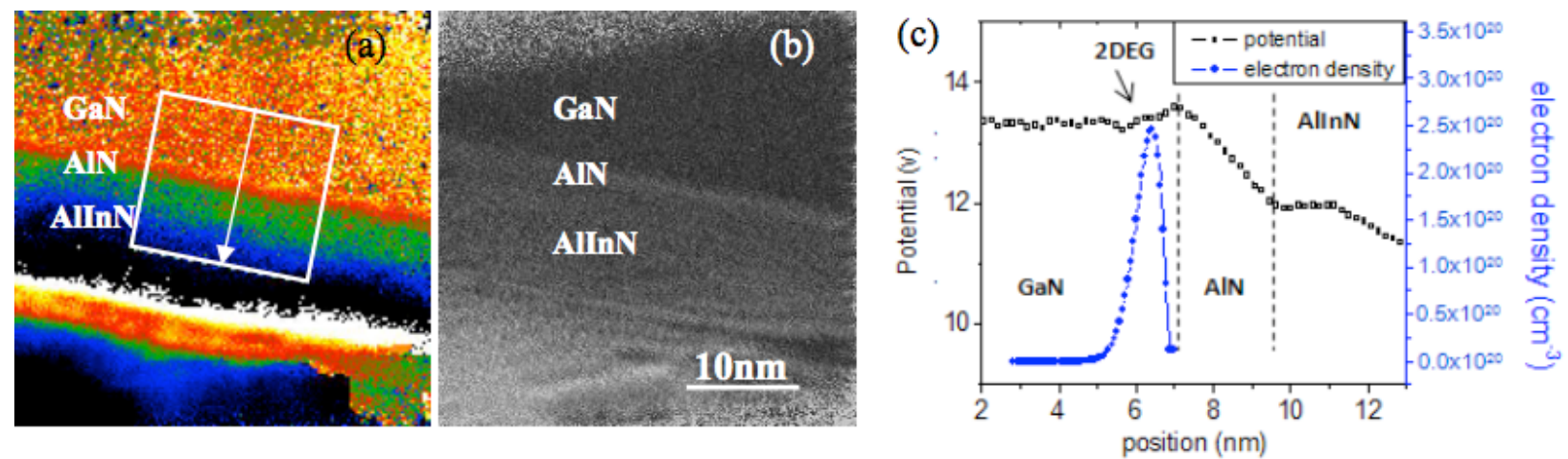

Figure 2. (a) Phase, and (b) amplitude, images of AlInN/AlN/GaN HEMT. (c) Potential profile (open squares) and electron distribution (filled circles) across interface region. $2 \mathrm{DEG} \sim 2.1 \times 10^{13} \mathrm{~cm}^{-2}[2]$.
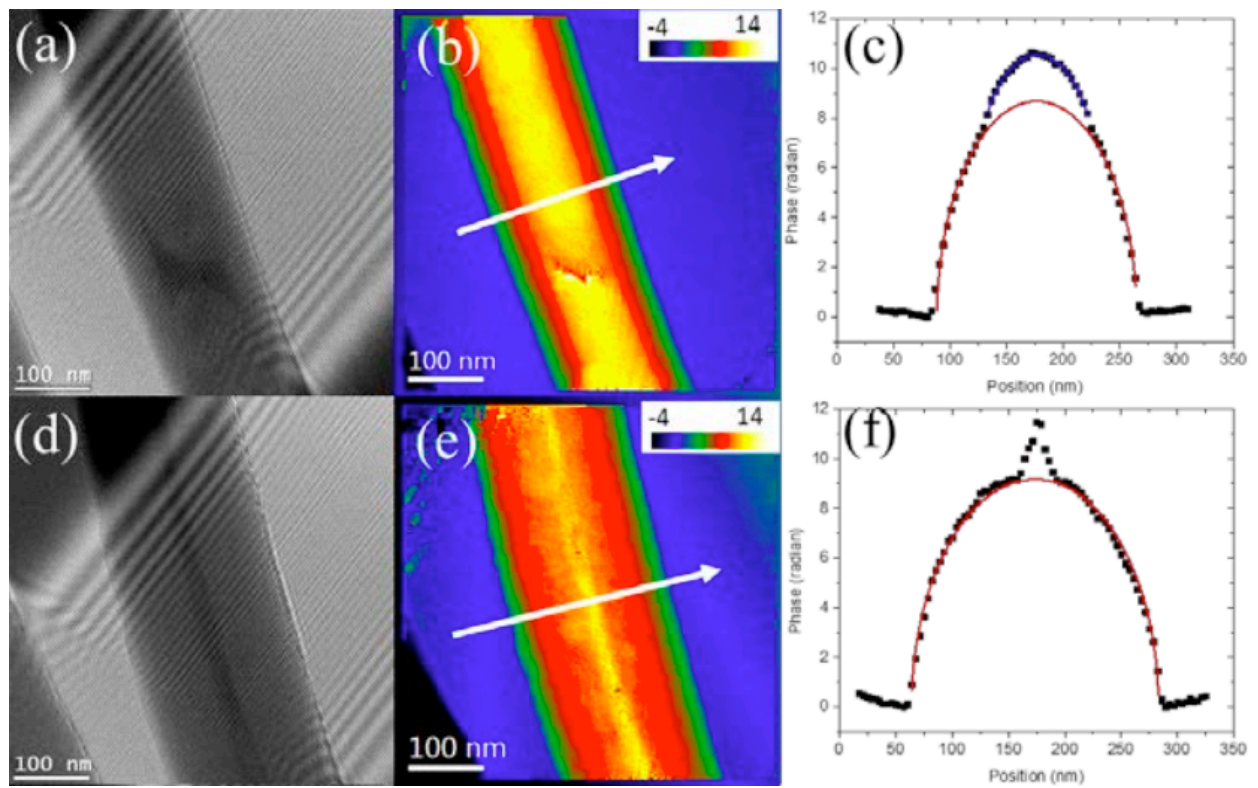

Figure 3. (a) and (d) Holograms of Ge/Lix Ge core/shell NW structure taken during lithiation; (b) and (e) Corresponding reconstructed phase images, shown in pseudo-color (scale bar shown at top right is in units of radian); (c) and (f) Phase profiles along the white arrows in (b) and (e), respectively [3]. 